\title{
Circulating RNAs, circ_4911 and circ_4302, are novel regulators of endothelial cell function under a hepatocellular carcinoma microenvironment
}

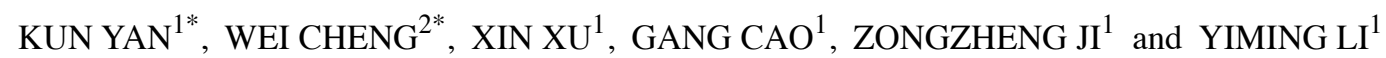 \\ ${ }^{1}$ Department of General Surgery, Second Affiliated Hospital of Xi'an Jiaotong University, Xi'an, Shaanxi 710004; \\ ${ }^{2}$ Department of Respiration, Xi'an Children Hospital, Xi'an, Shaanxi 710003, P.R. China
}

Received July 15, 2019; Accepted January 16, 2020

DOI: $10.3892 / o r .2020 .7702$

\begin{abstract}
Hepatocellular carcinoma (HCC) is a common hypervascular tumor disease. Endothelial cells, as a crucial component of the tumor microenvironment, have been reported to participate in angiogenesis and influence the development of tumors, including HCC. Recent studies have demonstrated that circulating RNAs (circRNAs) participate in the functional regulation of endothelial cells. However, the expression and function of circRNAs in endothelial cells under the HCC microenvironment is still unclear. In the present study, we analyzed the expression profiles and investigated the role of circRNAs in human umbilical vein endothelial cells (HUVECs) co-cultured with human primary hepatoma cells. Based on an RNA-sequencing assay, we screened 19 significantly downregulated circRNAs in HUVECs under an HCC microenvironment. Subsequently, we validated the expression of the candidate circRNAs using RT-qPCR, and selected two of the most downregulated circRNAs among them, circ_4911 and circ_4302. Next, through circRNA overexpression experiments, we demonstrated that overexpression of circ_4911 and circ_4302 both inhibited the proliferation and migration of HUVECs, and arrested cells at the GO/G1 stage, while promoting adhesion. Overall, in the present study, we identified the roles of circ_4911 and circ_4302 in regulating functions of HUVECs under an HCC microenvironment.
\end{abstract}

Correspondence to: Dr Kun Yan, Department of General Surgery, Second Affiliated Hospital of Xi'an Jiaotong University, 157 Xiwu Road, Xi'an, Shaanxi 710004, P.R. China

E-mail: kun_yyan@163.com

*Contributed equally

Key words: circRNA-sequencing, human umbilical vein endothelial cells, proliferation, migration, hepatocellular carcinoma, HCC microenvironment

\section{Introduction}

Worldwide, hepatocellular carcinoma (HCC) is considered as the most common type of hepatic malignancy, accounting for approximately $90 \%$ of all primary liver cancers (1). According to research data, HCC ranks fifth in incidence among the most common cancers in men and seventh in women (2). Unfortunately, the majority of patients are diagnosed at an advanced stage of HCC with metastasis and are not indicated for curative therapy, such as resection, transplantation or ablation (3). Moreover, HCC is also associated with the characteristics of rapid progression and a high rate of lethality. Therefore, to enhance the survival and improve the quality of life of HCC patients, research concerning the identification of novel targets and the development of new therapeutic strategies against $\mathrm{HCC}$ progression is urgently needed, especially concerning the metastasis of HCC (4).

Recent studies have demonstrated that $\mathrm{HCC}$ is a typical hypervascular tumor, with tissue composed of tumor cells and blood vessels $(5,6)$. Evidence has confirmed that angiogenesis is a key process closely related to tumor initiation and malignant evolution $(7,8)$. In addition, Sun and Liao also validated that physiological or pathological neovascularization is a necessary process in the initiation of tumor tissue ischemia, growth or metastasis (9). Hence, anti-angiogenic treatment has received much attention. Moreover, researchers have verified that tilting the balance toward stimulatory angiogenic factors is a crucial mechanism used by tumor cells to drive vascular growth and to obtain blood supply by attracting and activating cells from the tumor microenvironment (10). Furthermore, the endothelium consists of a typical and important type of stromal cells present in the tumor microenvironment. Studies have demonstrated that endothelial cells are recruited by cancer cells by secreting angiogenic factors to sustain tumor vascular networks (11). Moreover, tumor size and tumor angiogenesis could be reduced when this crosstalk is interrupted (12). Therefore, endothelial cells are not only a critical part of the tumor microenvironment of HCC; their association with cancer cells participates in the entire process of tumor angiogenesis (4). From this point of view, the dysfunction of endothelial cells in the tumor microenvironment has important effects on the progression and development of HCC. 
Recently, suggested as a subclass of endogenous non-coding RNAs existing in mammalian cells, circular RNAs (circRNAs) have emerged as new 'stars' of the non-coding RNA world. By virtue of the development of next-generation sequencing and bioinformatics technology, the increasing abundance of expression and biological function of circRNAs have been recognized (13). Additionally, circRNAs are considered to feature high cell-type/tissue/developmental specific expression and stable structure, and they mostly consist of exonic transcripts which are generated during the process of back splicing $(14,15)$. Researchers have found that circRNAs possess numerous properties conferred by their structure-a covalently closed continuous loop with the 3 ' and 5 'ends joined together (16). Recently, we have found that circRNAs are widely expressed and act as vital regulators in the process of transcriptional and post-transcriptional gene expression in human cells (17). Moreover, the relevance of circRNAs in multiple human cancers has been discovered, HCC included (18). The dominant role played by pathophysiological regulation of circRNAs has been verified by in-depth studies of circRNAs in the tumor field (19). In addition, recent studies have documented that circRNAs are involved in modulating the functions of stimulated endothelial cells (20-22). However, the expression and function of circRNAs in endothelial cells exposed to an HCC microenvironment remain unclear.

In the present study, we constructed a co-culture model of human primary hepatoma cells and umbilical vein endothelial cells. Based on this model, we determined the expression profile of circRNAs in endothelial cells under an HCC microenvironment by high throughput sequencing analysis. At the same time, a functional analysis and screening of differentially expressed circRNAs with significance were carried out. Moreover, we validated the effects of target circRNAs on endothelial cell function through overexpression experiments.

\section{Materials and methods}

Cell culture. The human umbilical vein and HCC tissues were obtained from the Northwest Women's and Children's Hospital (Shaanxi, China) from patients suffered who underwent surgery from March 2014 to February 2018 at the hospital. the average age of the patients was $57 \pm 8$ with the sex ratio at 3.83:1 (male vs. female). The primary human umbilical vein endothelial cells (HUVECs) and primary hepatoma cells (HPHCs) were extracted from these tissues. After tissue fragmentation, separation and purification, HUVECs were maintained in Dulbecco's modified Eagle's medium (DMEM; Sigma-Aldrich; Merck KGaA) supplemented with $10 \%$ growth factors (ATCC); HPHCs were cultured in DMEM, supplemented with $10 \%$ fetal bovine serum (FBS) (Thermo Fisher Scientific, Inc.). Cells that were passaged to the second or third generation were used for co-culture experiment. The Ethics Committee of Northwest Women and Children's Hospital provided approval for all procedures in the present study. Written informed consent was obtained from the patients and their families.

For the establishment of an indirect co-culture system, Transwell culture inserts in 6 -well plates $(0.4 \mu \mathrm{m}$ pore-size polyester membrane; Corning) were employed. Briefly, after trypsinization and re-suspension with complete DMEM at a density of $5 \times 10^{4}$ cells $/ \mathrm{ml}$, HPHCs were seeded in Transwell inserts at $5 \times 10^{4} \mathrm{cells} / \mathrm{ml}$, while HUVECs were plated on Bioflex plates. Subsequently, cells were independently incubated for $72 \mathrm{~h}$ to reach confluence, and then an additional $48 \mathrm{~h}$ indirect co-culture was performed by using shared culture media. Finally, HUVECs were cultured with the HPHC-conditioned media for another $48 \mathrm{~h}$.

RNA sequencing. HUVECs cultured under HCC microenvironment and normal conditions were both divided into three groups of sequencing samples. The whole sequencing process was completed in cooperation with Lianchuan Biotech. Briefly, the RiboMinus Eukaryote kit (Qiagen, Inc.) was used to remove rRNA from total RNA samples $(3 \mathrm{mg}$ ) ahead of constructing the RNA-seq libraries. In accordance with the manufacturer's protocol, we prepared the strand-specific RNA-seq libraries using NEBNext Ultra Directional RNA Library Prep kit for Illumina (NEB; New England BioLabs, Inc.). After fragmentation of ribosome-depleted RNA samples (50 ng), random hexamer primers were used for the synthesis of first- and second-strand complementary DNA (cDNA). To remove the RNA template strand and synthesize second-strand cDNA, dUTP mix was used. Subsequently, to repair the cDNA fragment ends, we treated samples with the End-It DNA End Repair kit (Epicentre ${ }^{\circledR}$ ), and then added an $\mathrm{A}$ at the $3^{\prime}$ ends of the DNA fragments by using Klenow followed by adaptor ligation. Next, cDNA products were purified with uracil DNA glycosylase (to remove the second-strand cDNA), and 13-16 cycles of PCR amplification were executed. Library quality control was monitored with a Bioanalyzer 2100 (Agilent). Finally, the sequencing process was completed using a HiSeq 2000 (Illumina) on a 100-bp paired-end run. We deposited the RNA-sequencing data in Gene Expression Omnibus (GEO) (https://www.ncbi.nlm. nih.gov/geo/) (accession code: GSE77661).

Gene Ontology (GO) categories and Kyoto Encyclopedia of Genes and Genomes (KEGG) pathway analyses. Based on the Wallenius non-central hyper-geometric distribution, we performed GO enrichment analysis on the circRNAs with the GO seq R packages (23). To detect the statistical enrichment of the circRNAs, KEGG pathway enrichment analysis was executed using KOBAS software (http://www.genome. $\mathrm{jp} / \mathrm{kegg} /)$.

Overexpression and transfection. The expression vector used in this study was pCDS-At which was purchased from BioVector NTCC Inc. The sequences of circ_4911 and circ_4302 were synthesized by TsingKe Biological Technology. The circRNAs were cloned into the pCDS-At vector to construct overexpression vectors. Transfection was achieved using Lipofectamine 2000 transfection reagent (Thermo Fisher Scientific, Inc.) according to the manufacturer's protocol. Briefly, the HCC-conditioned HUVECs were seeded at a concentration of $1 \times 10^{5}$ cells/well in 6-well culture plates. After plating for $24 \mathrm{~h}$, transfection was performed with pCDS-circ_4911 and pCDS-circ_4302 at concentrations of 1,2 and $4 \mu \mathrm{g} / \mathrm{ml}$ for $24 \mathrm{~h}$, respectively. After transfection, the cells were washed twice with PBS, cultured under regular conditions and used for experiments at $24 \mathrm{~h}$. 
Reverse transcription-quantitative polymerase chain reaction $(R T-q P C R)$. Following transfection, HUVECs overexpressing the target gene were collected for RT-qPCR. First, total RNAs from the cultured cells were extracted with Trizol reagent (Invitrogen; Thermo Fisher Scientific, Inc.). After RNA purification, the PrimeScript 1st strand cDNA Synthesis Kit (Takara) was used for first-strand cDNA generation with use of the ProFlex ${ }^{\mathrm{TM}} 3$ x 32-well PCR System (Applied Biosystems; Thermo Fisher Scientific, Inc.). Subsequently, the StepOne Real-Time PCR System (Applied Biosystems; Thermo Fisher Scientific, Inc.) and the SYBR Green PCR kit (Takara) were used simultaneously to measure target gene expression. GAPDH was employed as the internal control. The qPCR procedure was as follow: $1 \mathrm{~min}$ at $95^{\circ} \mathrm{C}, 20 \mathrm{sec}$ at $95^{\circ} \mathrm{C}$ and $10 \mathrm{sec}$ at $56^{\circ} \mathrm{C}$ and $15 \mathrm{sec}$ at $72^{\circ} \mathrm{C}$ for 35 cycles, and finally held at $4^{\circ} \mathrm{C}$. The fold change of target gene expression was assessed according to the $2^{-\Delta \Delta \mathrm{Cq}}$ method and normalized to that of GAPDH (24).

Cell proliferation assay. The 3-(4,5-dimethylthiazol-2-yl)-2,5-diphenyltetrazolium bromide (MTT) assay was performed to detect cell proliferation. The HCC-conditioned HUVECs were seeded in 96-well culture plates (Thermo Fisher Scientific, Inc.) at a concentration of $1 \times 10^{5}$ cells/well for a $24 \mathrm{~h}$ incubation. Then, to each well $5 \mu \mathrm{l}$ of $10 \mathrm{mg} / \mathrm{ml}$ MTT was added, and the plates were incubated for a further $4 \mathrm{~h}$ at $37^{\circ} \mathrm{C}$ followed by another incubation for at least $12 \mathrm{~h}$ with $1 \mathrm{M} \mathrm{NaOH}$ solution supplemented with $1 \%$ SDS. The final absorbance at $490 \mathrm{~nm}$ was measured with a microplate spectrophotometer (Bio-Tek Instruments, Inc.).

Transwell migration assay. For the detection of cell migration, a Transwell 24 -well chamber ( $8.0 \mu \mathrm{m}$ pore membranes; Corning) was used according to the manufacturer's instructions. Briefly, the upper chamber containing $100 \mu \mathrm{l}$ of serum-free medium was seeded with $1 \times 10^{5}$ cells per well, and the lower chamber contained $600 \mu \mathrm{l}$ of complete medium as the chemoattractant. Then, cells were incubated at $37^{\circ} \mathrm{C}$ for $24 \mathrm{~h}$. After that, cotton swabs were used to remove the cells which remained on the upper surface of the membrane, leaving the migrated cells on the lower surface. Next, the cells were fixed and stained with $4 \%$ paraformaldehyde and $0.1 \%$ crystal violet solution, respectively. Finally, the cells that passed through the filter were photographed with an inverted fluorescence microscope (magnification, x20).

Cell cycle distribution. To analyze cell cycle distribution, flow cytometry was utilized. Briefly, conditioned HUVECs at a concentration of $3 \times 10^{5}$ cells/well were plated in 6-well plates after transfection with the overexpression vector. Next, we digested the cells with $0.25 \%$ trypsin. After centrifugation, the cells were collected and resuspended with PBS solution. Then, the cells were fixed in pre-ice-cold $70 \%$ ethanol for $4 \mathrm{~h}$ at $4^{\circ} \mathrm{C}$, washed with PBS solution at least three times and digested for 30 min with RNase A (KeyGen Biotech Corp., Ltd.) at $37^{\circ} \mathrm{C}$. The cells were stained with $50 \mu \mathrm{g} / \mathrm{ml}$ propidium iodide (PI) (KeyGen Biotech Corp., Ltd.) for $30 \mathrm{~min}$ in the dark, and the stained cells were evaluated by flow cytometry using a FACSAria II flow cytometer (Becton Dickinson). The data are expressed as a proportion of the total and were analyzed by Flow Jo 7.6.2 (Tree Star, Inc.).
Western blot analysis. The primary antibodies (anti-EGFR (cat. no. 4267S), anti-p-EGFR (cat. no. 3777S), anti-p38 (cat. no. 8690S, anti-p-p38 (cat. no. 4511S), anti-cyclin D1 (cat. no. 55506S), anti-E-cadherin (cat. no. 3195S) and anti-GAPDH (cat. no. 5174S) were purchased from Cell Signaling Technology Inc. and diluted at 1:1,000, while the species-specific horseradish peroxidase-conjugated secondary antibody (cat. no. 7054S; Cell Signaling Technology) was diluted at 1:5,000. According to the manufacturer's instructions, the cells were washed with pre-ice-cold PBS solution (P1022-500; Beijing Solarbio Science \& Technology Co., Ltd.) and protein extraction was completed using RIPA lysis buffer (CW2334; CW Biotech). Next, the proteins were isolated by centrifugation at $15,000 \mathrm{x} \mathrm{g}$ and $4^{\circ} \mathrm{C}$ for $15 \mathrm{~min}$. Then, the concentration of protein was determined using the Pierce ${ }^{\mathrm{TM}}$ BCA Protein Assay kit (Thermo Fisher Scientific, Inc.). Proteins were separated by electrophoresis through 10\% SDS-PAGE gels (Life Technologies; Thermo Fisher Scientific, Inc.), and the protein lysates $(30 \mu \mathrm{g})$ were transferred onto PVDF membranes (Millipore). The membranes were blocked in 5\% non-fat dry milk and incubated at $4^{\circ} \mathrm{C}$ overnight with primary antibodies. Finally, the protein bands were visualized and quantified using Image Lab software (v3.0; Bio-Rad Laboratories, Inc.) and ImageJ software (v1.46; National Institutes of Health).

Statistical analysis. Each sequencing sample was divided into three groups, and all cellular experiments were repeated at least three times. Tukey's test was performed for multiple comparisons between groups and ANOVA analysis was conducted for multiple comparisons between individual groups. All the analyses were performed using SPSS v22.0 (IBM Inc.). GraphPad Prism 6 v6.06 (GraphPad Software) was used as the image processing software in this study. $\mathrm{P}<0.05$ was considered to indicate a statistically significant difference.

\section{Results}

Profiling of circRNAs in the individual-cultured HUVECs and HUVECs co-cultured with HPHCs. To investigate the circRNA expression profiles in HUVECs under HCC microenvironment, we divided the HPHC-co-cultured HUVECs $(\mathrm{H})$ and naive HUVECs (CO) into three groups. Next, the circRNA transcripts were characterized by using RNA-sequencing analyses. Every sample group was sequenced on an Illumina HiSeq sequencer. The analysis results showed that the numbers of novel splicing junctions between the $\mathrm{H}$ and $\mathrm{CO}$ groups were consistent (Fig. 1A). Through transcriptional analysis, we found that the transcriptional sources of circRNAs in cells of the treatment and control groups were basically the same: most of them were derived from exons; $\sim 6-8 \%$ of circRNAs were transcribed from introns; the intergenic circRNAs occupied $<0.5 \%$ of the transcript (Fig. 1B). Afterwards, we analyzed the GO enrichment of these circRNAs to further explore their potential functions. For biological processes, circRNAs were mainly enriched in five processes: 'regulation of transcription (DNA-templated)', 'intracellular protein transduction', 'viral processes', 'regulation of gene expression' and the 'MAPK cascade'. Concerning the cellular components, the enriched GO terms included 'membrane' and 'cytoplasm'. In addition, 

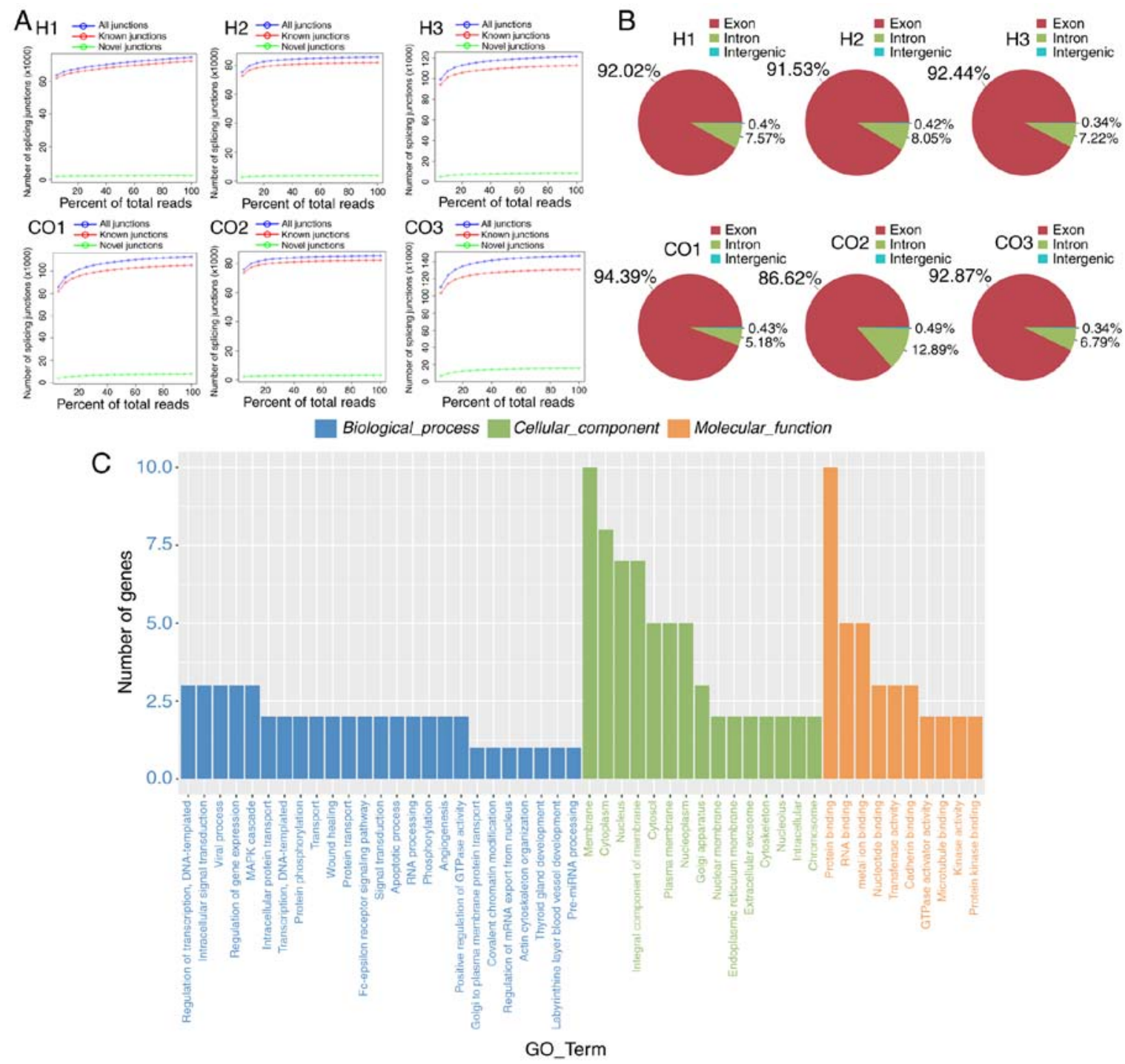

Figure 1. Profiling of circRNAs in HUVECs cultured individually and in co-culture with HPHCs. (A) Number of splice junctions per group. (B) Classification of the circRNA transcriptome in the treatment and control groups. (C) GO enrichment analysis presenting the number of circRNAs related to different biological processes, cellular components and molecular functions. HUVECs, human umbilical vein endothelial cells. HPHCs, human primary hepatoma cells. H, HPHC-co-cultured HUVECs; CO, naive HUVECs.

the circRNAs were predominately involved in the molecular function of 'protein binding' (Fig. 1C).

Identification and validation of differentially expressed circRNAs. Next, to identify differentially expressed circRNAs in HUVECs under HCC microenvironment, we profiled further analysis on profiling circRNA expression in $\mathrm{H}$ and $\mathrm{CO}$ groups. According to the volcano plot and differential circRNA expression enrichment plot, nineteen significant differentially expressed circRNAs were identified between the two groups (Fig. 2A and B). The heatmap displays the expression levels of these 19 circRNAs, and the results indicated that the expressions of circRNAs were significantly downregulated in the treatment group compared with the control, except circRNA-4289, circRNA-4382, circRNA-3702 and circRNA-3979 (Fig. 2C). Subsequently, GO categorization and KEGG pathway analyses were conducted to explore the putative function of these differentially expressed circRNAs. As shown in Fig. 2D, the most circRNA-related GO term was 'regulation of gene expression'.
For the pathway enrichment, circRNAs were mainly involved in the 'MAPK signaling pathway', 'ribosome biogenesis in eukaryotes' and 'lysine degradation' (Fig. 2D). Consistent with the sequencing results, the expression levels of all the circRNAs in HUVECs under the HCC microenvironment were significantly downregulated. To note, two most obviously down-modulated circRNAs in the $\mathrm{H}$ group vs. the $\mathrm{CO}$ group were circ_4911 and circ_4302 (Fig. 2E).

Circ_4911 affects cellular function and the expression of related proteins in HUVECs. Once circ_4911 was identified as a significantly downregulated gene in HUVECs under the action of HPHCs, our next step was to explore the effect of circ_4911 on endothelial cell function. To overexpress circ_4911, we used pCDS-At as the expression vector, and then we transfected the cultured HUVECs with pCDS-circ_4911 at concentrations of 1,2 and $4 \mu \mathrm{g} / \mathrm{ml}$. The expression of circ_4911 was significantly and dose-dependently increased in the circ_4911-overexpressing HUVECs (Fig. 3A). 


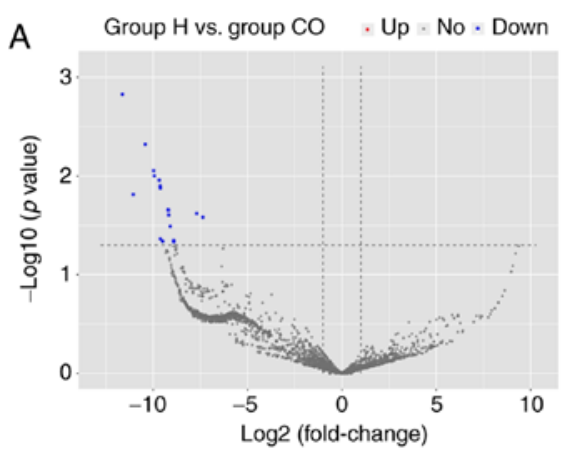

B Differentially expressed circRNAs in different groups

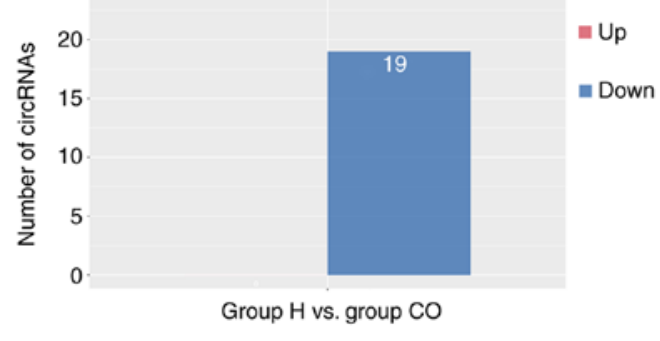

$\mathrm{C}$

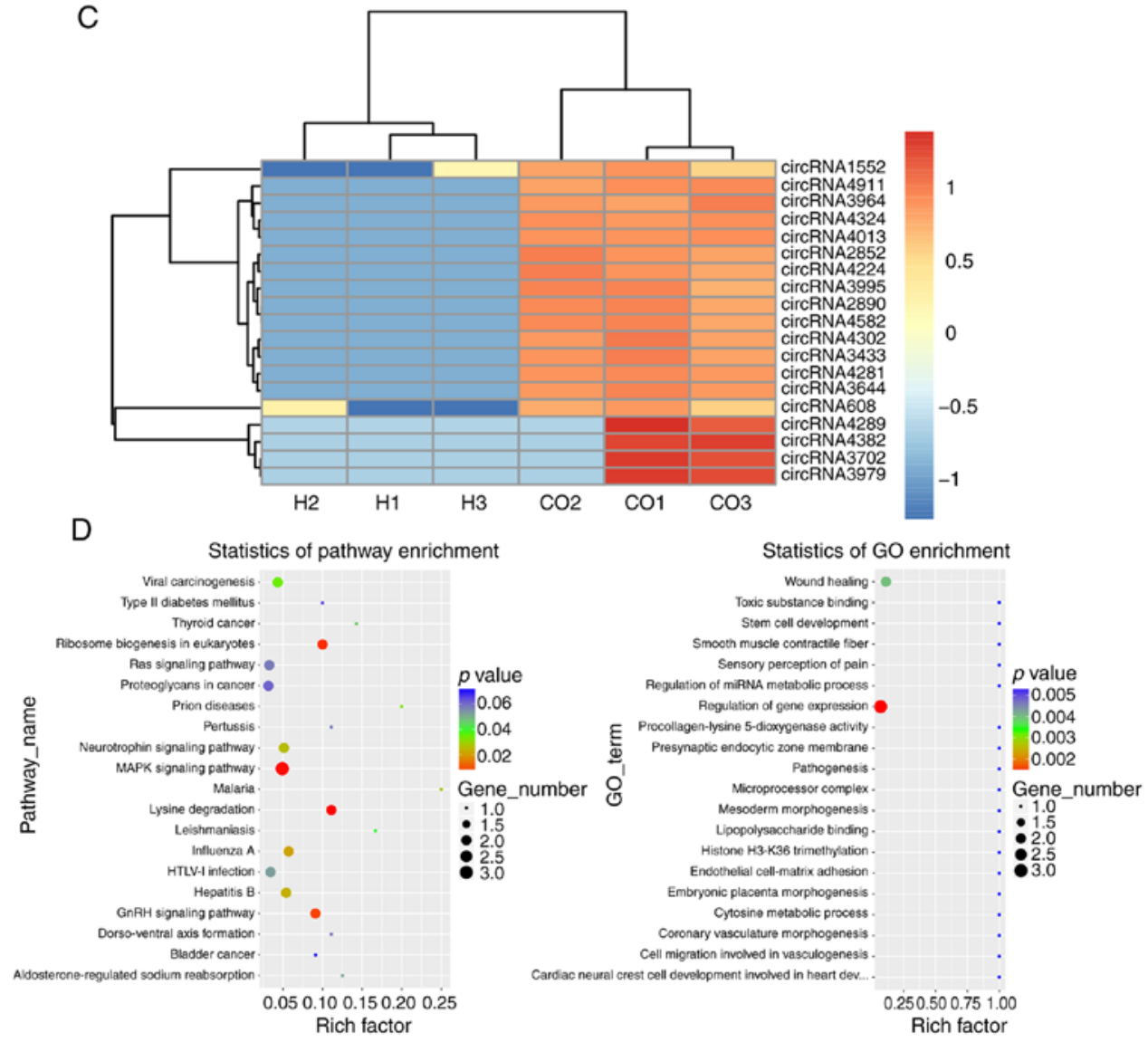

$\mathrm{E}$

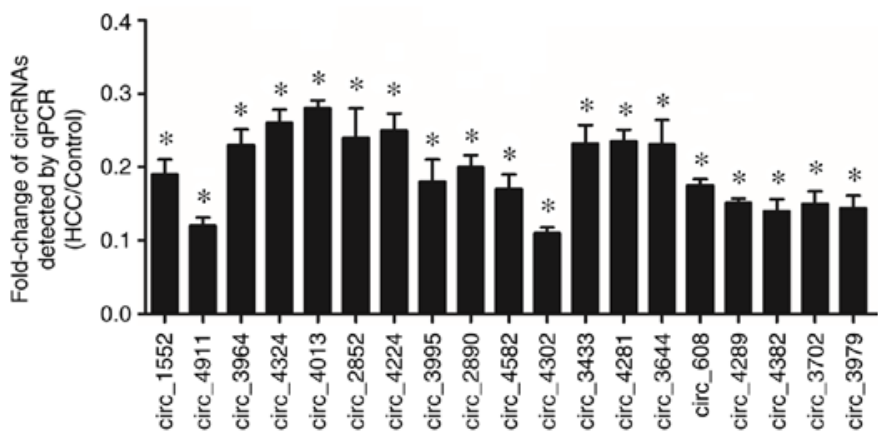

Figure 2. Identification and validation of the differentially expressed circRNAs. (A) Volcano plot showing the differential expression of circRNAs, standardized as $\mathrm{H}$ vs. CO. The vertical lines correspond to 2.0 -fold upregulation and downregulation, and the horizontal line represents a P-value of 0.05 . The blue and red points on the plot represent differentially expressed circRNAs with statistical significance. (B) The column plot presents the counts of differentially expressed circRNAs. (C) The heat map reflects the expression of circRNAs in every group. (D) Functional annotation clustering exhibiting the enrichment of circRNAs corresponding to different pathways and GO terms. (E) Fold-changes in the expression of circRNAs as detected by RT-qPCR. HUVECs, human umbilical vein endothelial cells. HPHCs, human primary hepatoma cells. H, HPHC-co-cultured HUVECs; CO, naive HUVECs.

Subsequently, the results of the cell proliferation assay showed that transfection with $4 \mu \mathrm{g} / \mathrm{ml}$ pCDS-circ_4911 significantly inhibited the proliferation activity of the HUVECs (Fig. 3B). Likewise, overexpression of circ_4911 significantly decreased 

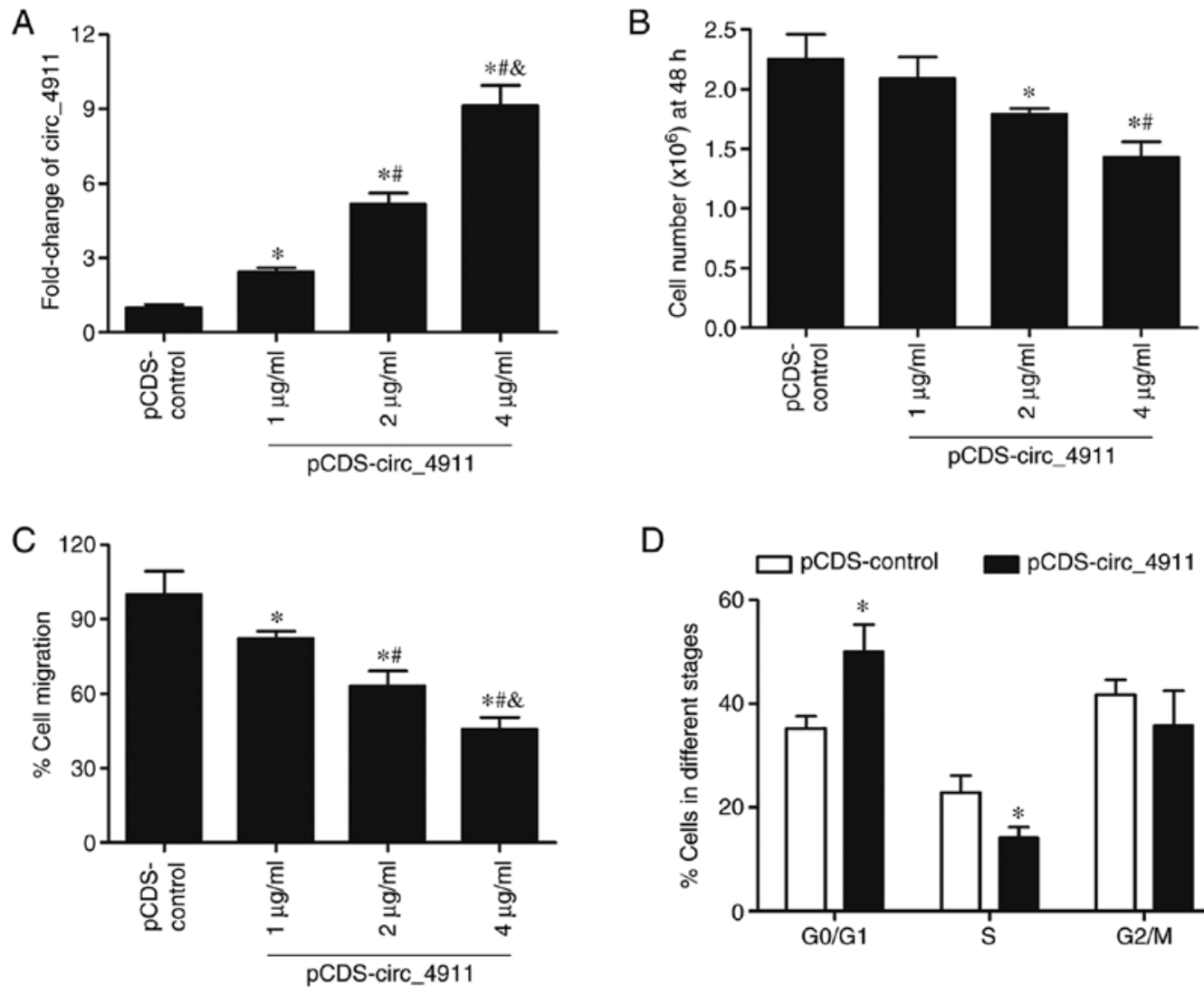

$\mathrm{D}$
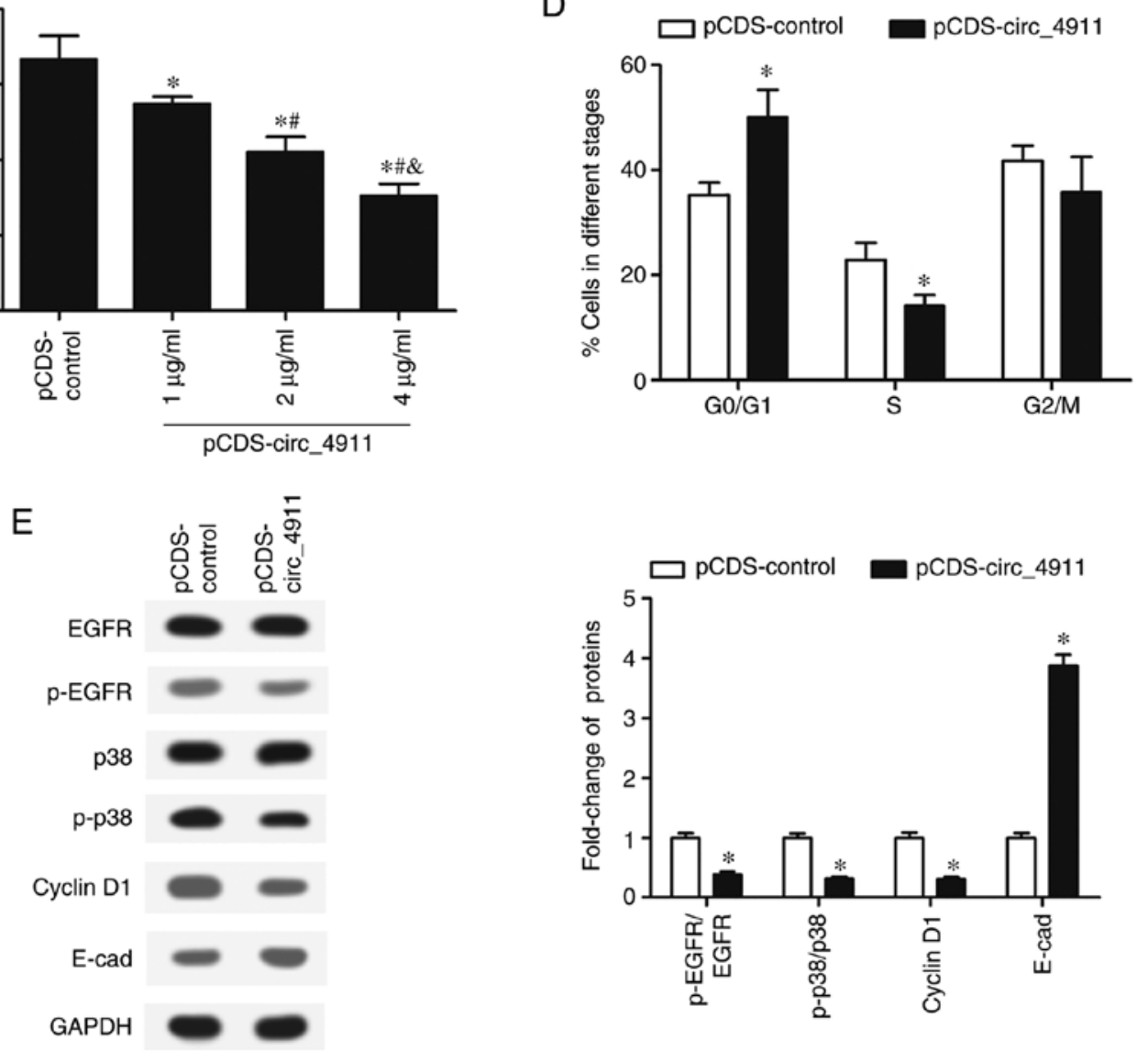

Figure 3. Circ_4911 affects the cellular function and expression of related factors in the HUVECs. Cultured HUVECs under HCC environment were transfected with the pCDS-circ_4911 expression vector at concentrations of 1,2 and $4 \mu \mathrm{g} / \mathrm{ml}$ for $24 \mathrm{~h}$. Then, the conditioned cells were used in follow-up experiments. (A) The fold change in circ_4911 expression was detected by RT-qPCR. (B) MTT assay showing the number of cells after $48 \mathrm{~h}$ of incubation. (C) The cell migration rate was measured by using the Transwell method. (D) Using flow cytometry, the proportions of cells in different stages of the cell cycle were analyzed. (E) Visualized and quantified results of target protein expression obtained by western blot analysis. Three independent experiments were carried out for each treatment. All data are presented as the mean \pm SEM. ${ }^{*} \mathrm{P}<0.05$ compared with the $\mathrm{pCDS}$ control group; ${ }^{\text {\& }} \mathrm{P}<0.05$ compared with the $2 \mu \mathrm{g} / \mathrm{ml}$ pCDS-circ_4911 group; ${ }^{\prime \prime} \mathrm{P}<0.05$ compared with the $1 \mu \mathrm{g} / \mathrm{ml}$ pCDS-circ_4911 group in A and C, and $2 \mu \mathrm{g} / \mathrm{ml}$ pCDS-circ_4911 group in B, respectively. HUVECs, human umbilical vein endothelial cells; HCC, hepatocellular carcinoma; E-cad, E-cadherin; EGFR, epidermal growth factor receptor.

the migration rate of HUVECs in a dose-dependent manner (Fig. 3C). In addition, circ_4911 also modulated the cellular cycle of HUVECs; there was a significant elevation in the proportion of cells in the G0/G1 stages when circ_4911 was overexpressed, while the proportion of cells in the S stage was declined; the ratio of cells in the G2/M stage was not significantly affected by circ_4911 overexpression (Fig. 3D). To further validate the effects of circ_4911 overexpression on cell function, we detected the expression levels of several key proteins related to the cell cycle, proliferation, migration and adhesion. The visualized and quantified results of the western blot analysis indicated that circ_4911 overexpression significantly suppressed the activation of epidermal growth factor receptor (EGFR) and p38 and the expression of cyclin D1, while it greatly increased the expression of E-cadherin (Fig. 3E). In general, the expression of circ_4911 was found to play an inhibitory role in the proliferation and migration of HUVECs. 

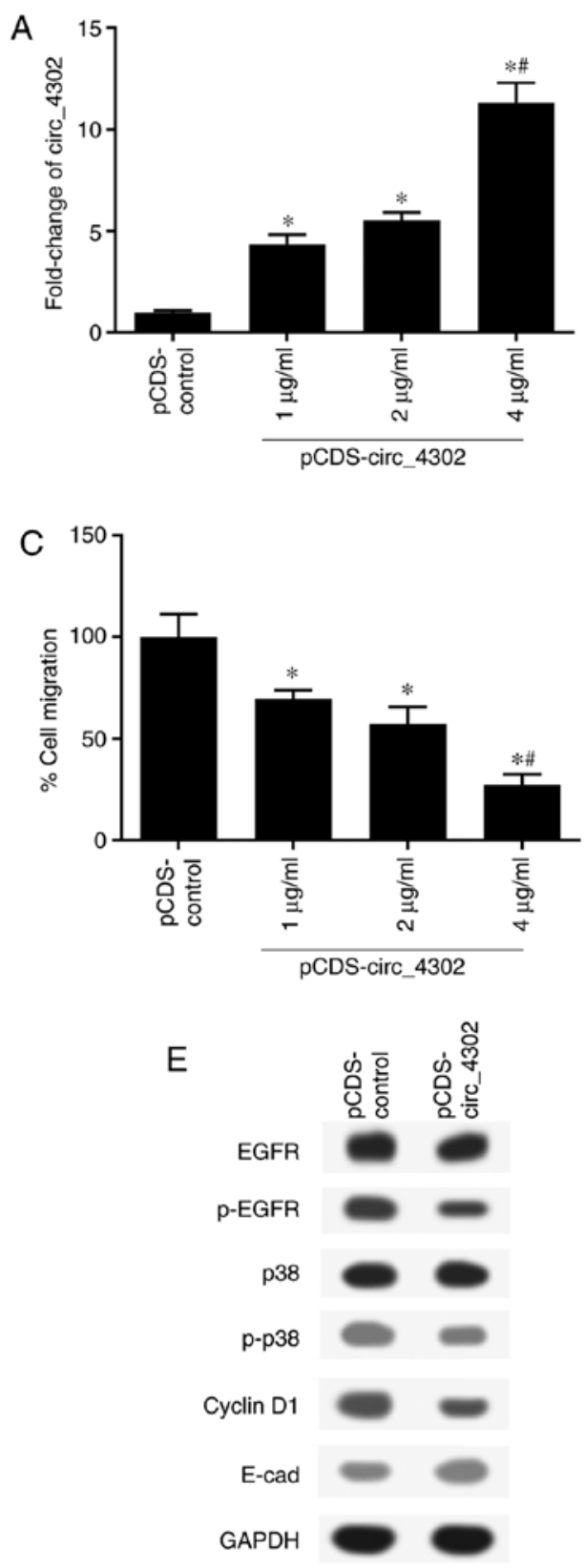

B

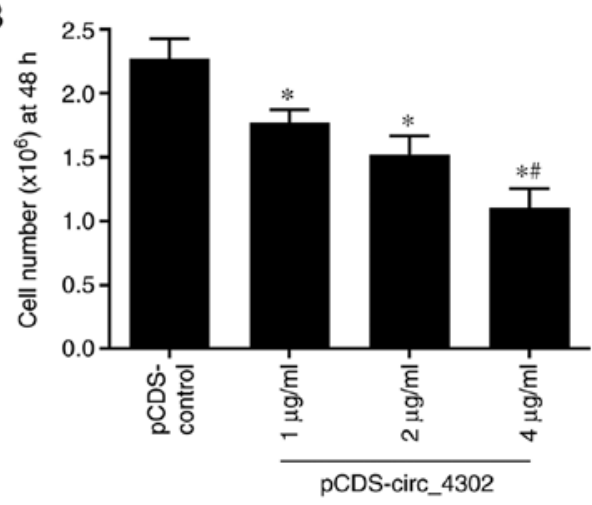

D $\square$ pCDS-control $=$ pCDS-circ_4302
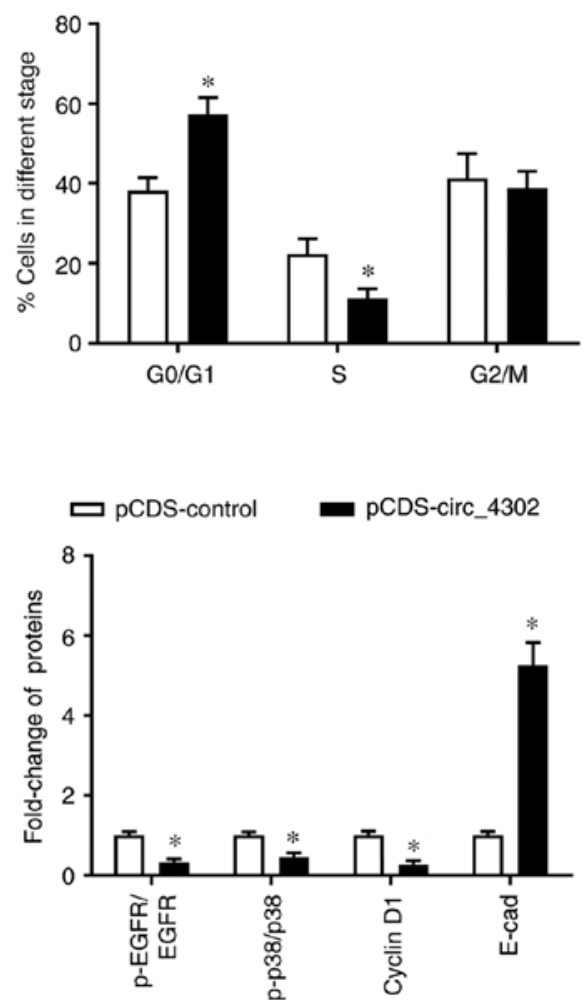

Figure 4. Circ_4302 plays a regulatory role in cellular function and the expression of related factors in HUVECs. A 24 h transfection was performed on HCC-conditioned HUVECs using the pCDS-circ_4302 expression vector at concentrations of 1,2 and $4 \mu \mathrm{g} / \mathrm{ml}$. Then, the cultured cells were used for the following experiments. (A) The expression level of circ_4302 was detected by RT-qPCR. (B) The number of living cells was detected after $48 \mathrm{~h}$ of incubation using the MTT assay. (C) The Transwell method was performed to measure the cell migration rate. (D) Analysis of the proportions of cells in different stages of the cycle was conducted using flow cytometry. (E) Western blot assay was used to visualize and quantify target protein expression. All data are presented as the mean \pm SEM of three independent experiments. ${ }^{*} \mathrm{P}<0.05$ vs. the pCDS-control group. ${ }^{*} \mathrm{P}<0.05$ compared with the $2 \mu \mathrm{g} / \mathrm{ml}$ pCDS-circ 4302 group. HUVECs, human umbilical vein endothelial cells; HCC, hepatocellular carcinoma; E-cad, E-cadherin; EGFR, epidermal growth factor receptor.

Circ_4302 plays a regulatory role in cellular function and related protein expression of HUVECs. Circ_4302 was another circRNA selected as being markedly downregulated in the HUVECs. To verify the function exerted by circ_4302 on cellular events in HUVECs, we carried out overexpression experiments as for circ_4911. First, the results of RT-q PCR confirmed the effect of transfection with the pCDS-circ_4302 vector (Fig. 4A). Then, compared with the control group, the cell viability of the HUVECs significantly declined after the transfection with pCDS-circ_4302 (Fig. 4B). Additionally, the cell migration rate of HUVECs was restrained by circ_4302 overexpression vector in a dose-dependent manner (Fig. 4C).
According to the experiments above, we found that the transfection effect at the concentration of $4 \mu \mathrm{g} / \mathrm{ml}$ was the most obvious. Thus, we used $4 \mu \mathrm{g} / \mathrm{ml}$ pCDS-circ_4302 in subsequent experiments. Consistent with the effect exerted by circ_4911 overexpression on the cell cycle, overexpressing of circ_4302 similarly arrested HUVECs at the G0/G1 stage (Fig. 4D). Moreover, the expression of p-EGFR, p-p38 and cyclin D1 was obviously decreased in the HUVECs overexpressing circ_4302, while that of E-cadherin was significantly enhanced (Fig. 4E). Taking together, circ_4302 had the same regulatory effects as circ_4911 in regards to proliferation, migration and adhesion of HUVECs. 


\section{Discussion}

In recent years, sequencing and biotechnology have become important technologies with great promise in biological research. RNA-sequencing, also called transcriptome sequencing technology, is an emerging technology based on high-throughput sequencing and reflects the expression levels of RNAs (small RNAs and non-coding RNAs included) in conditioned samples. In the present study, we analyzed and predicted the distribution, expression and function of circRNAs in human umbilical vein endothelial cells (HUVECs) under a HCC microenvironment through the use of RNA-sequencing.

CircRNAs, as a special type of non-coding RNA molecule, have emerged as one of the latest research 'hotspots' in the field of RNA. In the early years, RNA splicing errors, transcriptional noise and RT-PCR artefacts were once the labels of circRNAs (25). However, researchers have since discovered that circRNAs have widely enriched expression, as well as transcriptional and post-transcriptional regulatory functions in human cells (17). Furthermore, studies have demonstrated that circRNAs are expressed in a tissue- and cell-specific manner (15). In this study, we analyzed the circRNA expression profile in human endothelial cells under an HCC microenvironment by utilizing circRNA microarray analysis. The results indicated abundant expression of circRNAs in HUVECs, and the majority were derived from exon circularization. Moreover, these circRNAs were predicted to be associated with a variety of cellular components, biological processes and molecular functions.

In-depth studies provided evidence that circRNAs are expressed at significantly different levels in stimulated HUVECs and normal HUVECs, suggesting that circRNAs play a specific role in endothelial cells. Analysis of the expression profiles of circRNAs in HUVECs stimulated by ox-LDL revealed 943 circRNAs that were differentially expressed with two-fold changes; among them, hsa_circ_0003575 was upregulated dramatically in ox-LDL-induced HUVECs (21). The expression of cZNF292 was found to be significantly upregulated among the circRNAs screened from hypoxia-induced HUVECs with differential expression (20). Similarly, researchers found a variety of circRNAs that were differentially expressed in HUVECs exposed to hyperglycaemia (16). In the present study, by analyzing the expression of circRNAs in human primary hepatoma cells (HPHCs) co-cultured with HUVECs, we found 19 circRNAs that displayed significant downregulation compared with the control group. In addition, using RT-qPCR analysis, we verified the dysregulation of these circRNAs, and screened out two circRNAs with the most significant differential expression: circ_4911 and circ_4302.

To further explore the roles that circ_4911 and circ_4302 play in the functions of HUVECs, we overexpressed circ_4911 and circ_4302 in HUVECs. The results led us to the following discovery. Overexpression of circ_4911 and circ_4302 significantly inhibited the proliferation and migration of HUVECs, and arrested cells at the GO/G1 stage thus affecting cell replication. The expression levels of key proteins related to these cell events were also repressed. In addition, the upregulation of E-cadherin expression demonstrated that circ_4911 and circ_4302 could promote cell adhesion of HUVECs. In previous studies, circRNAs was proven as crucial molecular regulators of cell function in endothelial cells.
Dang and colleagues reported that knockdown of circRNA hsa_circ_0010729 enhanced apoptosis, and could reverse hypoxia-induced inhibition of proliferation and migration in HUVECs (19). Under ox-LDL stimulation, hsa_circ_0003575 silencing was demonstrated to promote proliferation, increase the migration distance and enhance angiogenesis in HUVECs, while apoptosis was decreased (21). Hsa_circRNA-0054633 was validated to have protective effects against endothelial cell dysfunction stimulated by high glucose (26).

Previously, endothelial cells were considered as one of the key components in the tumor microenvironment, and they were found to play an irreplaceable role in the development and progression of tumors (27). More importantly, the proliferation and migration of endothelial cells are involved in the angiogenesis of tumors (11). It has been found that tumor cells recruit endothelial cells and stimulate cell proliferation and migration of endothelial cells by secreting signal factors, while reducing cell adhesion, so as to maintain the vascular network of tumors and ensure the growth of tumors $(4,28,29)$. In this study, differentially expressed circ_4911 and circ_4302 were selected from HUVECs in an HCC microenvironment, and both were proven to play key roles in the cell cycle, proliferation, migration and adhesion of endothelial cells. We proposed that circ_4911 and circ_4302 are crucial molecules in the development of HCC that may provide new targets for HCC therapy.

In conclusion, our study analyzed the expression profile of circRNAs in HUVECs in an HCC microenvironment and identified two significantly downregulated circRNAs: circ_4911 and circ_4302. Moreover, through overexpression experiments, we demonstrated that circ_4911 and circ_4302 regulate the proliferation, migration and adhesion of endothelial cells. Therefore, we propose that circ_4911 and circ_4302 may play important roles in the progression and development of HCC. However, there are also limitations in the present study. The abnormal expression of circ_4911 and circ_4302 should be identified in tissues, and also the correlation between circRNAs and the clinical characteristics of HCC patients should be analyzed. Moreover, further research will aim to illustrate the potential molecular mechanism of circ_4911 and circ_4302 in regulating the processes of endothelial cells under an HCC microenvironment, based on classical signaling pathways. These findings may provide novel targets for HCC diagnosis and therapy.

\section{Acknowledgements}

Not applicable.

\section{Funding}

The present study was funded by the Science and Technology Research and Development Program of Shaanxi Province (2011K13-03-11), the Science and Technology Project of Xi'an [SF1203 (3)], and the State Scholarship Fund of China (No. 01806285146).

\section{Availability of data and materials}

The datasets used and/or analyzed during the present study are available from the corresponding author on reasonable request. 


\section{Authors' contributions}

KY and WC made substantial contributions to the design of the present study. Data acquisition and interpretation were conducted by KY, WC, XX, GC and YL. KY and ZJ wrote the manuscript. WC and $\mathrm{ZJ}$ critically revised the manuscript for important intellectual content. All authors approved the final version of the manuscript. All authors read and approved the manuscript and agree to be accountable for all aspects of the research in ensuring that the accuracy or integrity of any part of the work are appropriately investigated and resolved.

\section{Ethics approval and consent to participate}

The experimental protocol of this study was approved by the Committee on the Second Affiliated Hospital of Xi'an Jiaotong University (Shaanxi, China). Written informed consent was obtained from each participant prior to tissue collection.

\section{Patient consent for publication}

Not applicable.

\section{Competing interests}

The authors declare that they have no competing interests.

\section{References}

1. Siegel RL, Miller KD and Jemal A: Cancer statistics, 2018. CA Cancer J Clin 68: 7-30, 2018

2. Torre LA, Bray F, Siegel RL, Ferlay J, Lortet-Tieulent J and Jemal A: Global cancer statistics, 2012. CA Cancer J Clin 65 87-108, 2015.

3. Sun HZ, Song YL and Wang XY: Effects of different anesthetic methods on cellular immune and neuroendocrine functions in patients with hepatocellular carcinoma before and after surgery. J Clin Lab Anal 30: 1175-1182, 2016.

4. Feng T, Yu H, Xia Q, Ma Y, Yin H, Shen $Y$ and Liu $X$ Cross-Talk mechanism between endothelial cells and hepatocellular carcinoma cells via growth factors and integrin pathway promotes tumor angiogenesis and cell migration. Oncotarget 8 : 69577-69593, 2017.

5. Tomizawa M, Kondo F and Kondo Y: Growth patterns and interstitial invasion of small hepatocellular carcinoma. Pathol Int 45: 352-358, 1995.

6. Yukawa H, Suzuki K, Aoki K, Arimoto T, Yasui T, Kaji N, Ishikawa T, Ochiya T and Baba Y: Imaging of angiogenesis of human umbilical vein endothelial cells by uptake of exosomes secreted from hepatocellular carcinoma cells. Sci Rep 8: 6765, 2018.

7. Park JH, Kim SH, Choi MC, Lee J, Oh DY, Im SA, Bang YJ and Kim TY: Class II histone deacetylases play pivotal roles in heat shock protein 90-mediated proteasomal degradation of vascular endothelial growth factor receptors. Biochem Biophy Res Commun 368: 318-322, 2008.

8. Zhao Y and Adjei AA: Targeting angiogenesis in cancer therapy: Moving beyond vascular endothelial growth factor. Oncologist 20: 660-673, 2015.

9. Sun J and Liao JK: Induction of angiogenesis by heat shock protein 90 mediated by protein kinase akt and endothelial nitric oxide synthase. Arterioscler Thromb Vasc Biol 24: 2238-2244, 2004.
10. Weis SM and Cheresh DA: Tumor angiogenesis: Molecular pathways and therapeutic targets. Nat Med 17: 1359-1370, 2011.

11. Manzi M, Bacigalupo ML, Carabias P, Elola MT, Wolfenstein-Todel C, Rabinovich GA, Espelt MV and Troncoso MF: Galectin-1 controls the proliferation and migration of liver sinusoidal endothelial cells and their interaction with hepatocarcinoma cells. J Cell Physiol 231: 1522-1533, 2016.

12. Vasudev NS and Reynolds AR: Erratum to: Anti-Angiogenic therapy for cancer: Current progress, unresolved questions and future directions. Angiogenesis 17: 495-497, 2014.

13. Zheng XB, Zhang M and Xu MQ: Detection and characterization of ciRS-7: A potential promoter of the development of cancer. Neoplasma 64: 321-328, 2017.

14. Szabo L and Salzman J: Detecting circular RNAs: Bioinformatic and experimental challenges. Nat Rev Genet 17: 679-692, 2016.

15. Yao T, Chen Q, Fu L and Guo J: Circular RNAs: Biogenesis, properties, roles, and their relationships with liver diseases. Hepatol Res 47: 497-504, 2017.

16. Shang FF, Luo S, Liang X and Xia Y: Alterations of circular RNAs in hyperglycemic human endothelial cells. Biochem Biophys Res Commun 499: 551-555, 2018.

17. Hou LD and Zhang J: Circular RNAs: An emerging type of RNA in cancer. Int J Immunopathol Pharmacol 30: 1-6, 2017.

18. Fu L, Yao T, Chen Q, Mo X, Hu Y and Guo J: Screening differential circular RNA expression profiles reveals hsa_circ_0004018 is associated with hepatocellular carcinoma. Oncotarget 8: 58405-58416, 2017.

19. Dang RY, Liu FL and Li Y: Circular RNA hsa_circ_0010729 regulates vascular endothelial cell proliferation and apoptosis by targeting the miR-186/HIF-1 $\alpha$ axis. Biochem Biophys Res Commun 490: 104-110, 2017.

20. Jes-Niels B, Nicolas J, Heumüller AW, Chen W, Boon RA, Stellos K, Zeiher AM, John D, Uchida S and Dimmeler S: Identification and characterization of hypoxia-regulated endothelial circular RNA. Circ Res 117: 884-890, 2015.

21. Li CY, Ma L and Yu B: Circular RNA hsa_circ_0003575 regulates oxLDL induced vascular endothelial cells proliferation and angiogenesis. Biomed Pharmacother 95: 1514-1519, 2017.

22. Li J, Li Z, Jiang P, Peng M, Zhang X, Chen K, Liu H, Bi H, Liu X and Li X: Circular RNA IARS (circ-IARS) secreted by pancreatic cancer cells and located within exosomes regulates endothelial monolayer permeability to promote tumor metastasis. J Exp Clin Cancer Res 37: 177, 2018.

23. Young MD, Wakefield MJ, Smyth GK and Oshlack A: Gene ontology analysis for RNA-seq: Accounting for selection bias. Genome Biol 11: R14, 2010.

24. Livak KJ and Schmittgen TD: Analysis of relative gene expression data using real-time quantitative PCR and the 2(-Delta Delta C(T)) method. Methods 25: 402-408, 2001.

25. Zhao W, Cheng Y, Zhang C, You Q, Shen X, Guo W and Jiao Y: Genome-Wide identification and characterization of circular RNAs by high throughput sequencing in soybean. Sci Rep 7: 5636, 2017.

26. PanL,LianW,Zhang X,HanS,CaoC,LiXandLiM:Humancircular RNA-0054633 regulates high glucose-induced vascular endothelial cell dysfunction through the microRNA-218/roundabout 1 and microRNA-218/heme oxygenase-1 axes. Int J Mol Med 42: 597-606, 2018.

27. Brenner W, Beitz S, Schneider E, Benzing F, Unger RE, Roos FC, Thüroff JW and Hampel C: Adhesion of renal carcinoma cells to endothelial cells depends on PKCmu. BMC Cancer 10: 183, 2010.

28. Meng J, Liu Y, Han J, Tan Q, Chen S, Qiao K, Zhou H, Sun T and Yang C: Hsp90 $\beta$ promoted endothelial cell-dependent tumor angiogenesis in hepatocellular carcinoma. Mol Cancer 16: 72, 2017.

29. Choi H and Moon A: Crosstalk between cancer cells and endothelial cells: Implications for tumor progression and intervention. Arch Pharm Res 41: 711-724, 2018. 